\title{
Adoption and Implementation of the Surgical Safety Checklist: Improving Safety in an Italian Teaching Hospital
}

American Journal of Medical Quality I

(C) The Author(s) 2018

Reprints and permissions:

sagepub.com/journalsPermissions.nav DOI: 10.1 I77/10628606/8780354 ajmq.sagepub.com
To the Editor:

Although it is known that clinical risk management tools such as the Surgical Safety Checklist lead to greater safety for patients and protection for the operator, clinical risk management units have much work to do to implement and spread the use of quality health care tools.

In Southern Italy, the regional Clinical Risk Board, together with the clinical risk unit of Bari Policlinico ( $>1500$ beds), conducted an experimental survey with the main objective of monitoring correct utilization of the safety checklist during major surgeries.

In the operating room (OR), communication gaps between health operators can cause damage to patients. ${ }^{1}$ Despite the importance communication has gained in daily clinical practice, information flow is often lacking and can cause the omission and the leakage of relevant clinical news.

In 2004, the World Health Organization launched the Safe Surgery Saves Life campaign to standardize the communication process in the OR. The results of this campaign are still ambiguous today. ${ }^{2}$

The first part of study recruitment was retrospective, evaluating clinical records of surgery patients who had been discharged before the beginning of the initiative. The second part involved patients on the same unit for the same surgical procedures after monitoring began.

Comparing the results in the pre and post samples, we saw a sharp increase in the number of procedures where the surgical room checklist was completed (from $20.40 \%$ pre to 43.75 post) and, above all, a significant decrease in absent checklists (from $29.10 \%$ to $3.75 \%$ ). Although these results show improvement, they are still far below the safety range, for which completeness is about $80 \%$ to $100 \%$.

Applying methodologies and directives from clinical risk management, we demonstrated that it is possible to increase health operators' compliance in a surgical team using a simple and free intervention. At the same time, it was possible to increase the general perception of the evaluation of the hospital and patient safety scale, which represents some elements used to evaluate the safety culture of a sanitary system. ${ }^{3}$
The introduction of simple monitoring of the safety checklist enabled the prevention of clinical risk and protects the operator from medical appeals, which are increasingly frequent, and affects the decision to adopt defensive medical practices, which are a significant factor in the cost of care without a real benefit to patients. ${ }^{4}$

The involvement of the staff in safety problems should be systematic; the presence of a common language, continuing education, and personal awareness are fundamental instruments for pursuing this purpose. Moreover, it is necessary to comprehend the real importance and power of the Surgical Safety Checklist to avoid a superficial completion, without really verifying all the steps, just to perform a bureaucratic duty.

The challenge of the checklist is essentially cultural: its adoption should not be seen as an additional new administrative and bureaucratic duty but as an opportunity to provide safer care to patients, limiting the risk of error.

$$
\begin{array}{r}
\text { Davide Ferorelli, MD } \\
\text { Crudele Lucilla, MD } \\
\text { Leonardo Vincenti, MD } \\
\text { Fiorenza Zotti, PhD } \\
\text { Alessandro Dell'Erba, MD, PhD } \\
\text { Policlinico di Bari, Bari, Italy }
\end{array}
$$

\section{References}

1. Myers B, Mitchell C, Whitty JA, Donovan P, Coombes I. Prescribing and medication communication on the posttake ward round. Intern Med J. 2017;47:454-457.

2. Zingiryan A, Paruch JL, Osler TM, Hyman NH. Implementation of the surgical safety checklist at a tertiary academic center: Impact on safety culture and patient outcomes. Am J Surg. 2017;214:193-197.

3. McMullan SP, Thomas-Hawkins C, Shirey MR. Certified registered nurse anesthetist perceptions of factors impacting patient safety. Nurs Adm Q. 2017;41(1):56-69.

4. Panella M, Rinaldi C, Leigheb F, et al. Prevalence and costs of defensive medicine: a national survey of Italian physicians [published online January 1, 2017]. J Health Serv Res Policy. doi:10.1177/1355819617707224. 